\title{
Numerical solution of the two-dimensional Gross-Pitaevskii equation for trapped interacting atoms
}

\author{
Sadhan K. Adhikari \\ Instituto de Física Teórica, Universidade Estadual Paulista, 01.405-900 São Paulo, São Paulo, Brazil
}

(October 29, 2018)

\begin{abstract}
We present a numerical scheme for solving the time-independent nonlinear Gross-Pitaevskii equation in two dimensions describing the Bose-Einstein condensate of trapped interacting neutral atoms at zero temperature. The trap potential is taken to be of the harmonic-oscillator type and the interaction both attractive and repulsive. The Gross-Pitaevskii equation is numerically integrated consistent with the correct boundary conditions at the origin and in the asymptotic region. Rapid convergence is obtained in all cases studied. In the attractive case there is a limit to the maximum number of atoms in the condensate.
\end{abstract}

Accepted in Physics Letters A

Recently, there have been experiments [1] of BoseEinstein condensation in dilute bosonic atoms (alkali and hydrogen atoms) employing magnetic traps at ultra-low temperatures. These experiments have intensified theoretical investigations on various aspects of the condensate [2]1. The condensate can consist of few thousand to millions of atoms confined by the trap potential. The properties of the condensate at zero temperature are usually described by the nonlinear time-independent mean-field Gross-Pitaevskii (GP) equation [8], which properly incorporates the trap potential as well as the interaction among the atoms. The effect of the interaction leads to a nonlinear term in the GP equation which complicates its solution procedure.

There have been a series of recent studies which deal with the numerical solution of the three-dimensional GP equation [3 6]. These works have emphasized the serious difficulties in obtaining numerical convergence of the solution. There has been no such systematic study on the numerical solution of the GP equation in two dimensions. Although, there has been no experiments on BoseEinstein condensation of two-dimensional systems, this is a problem of great interest. A system of ideal Bose gas in two dimensions does not undergo condensation at a finite temperature [9]. However, condensation can take place under the action of a trap potential [10]. In order to achieve two-dimensional Bose-Einstein condensation in real three-dimensional traps, one should choose the frequency $\omega_{x y}$ in the $x-y$ plane negligibly small compared to that in the $z$ direction $\omega_{z}$ [11]. Also, there has been consideration of Bose-Einstein condensation in low-dimensional systems for particles confined by gravitational field or by a rotational container 12]. Possible experimental configurations for Bose-Einstein condensation in spin-polarized hydrogen in two dimensions are currently being discussed [2.77. Because of these interests, here we perform a critical study of the numerical solution of the time-independent GP equation in two dimensions for an interacting Bose gas under the action of a harmonic-oscillator-type trap potential. The interatomic interaction is taken to be both attractive and repulsive in nature.

In steady state at zero temperature the condensate wave function is described by the following effective nonlinear Schrödinger-type equation known as the GrossPitaevskii equation [8] for condensed neutral bosons in a harmonic trap:

$$
\left[-\frac{\hbar^{2}}{2 m} \nabla^{2}+\frac{1}{2} m \omega^{2} r^{2}+g \Psi^{2}(\mathbf{r})-\mu\right] \Psi(\mathbf{r})=0 .
$$

Here $\Psi(\mathbf{r})$ is the condensate wave function at position $\mathbf{r}$, $m$ the mass of a single bosonic atom, $m \omega^{2} r^{2} / 2$ the attractive harmonic-oscillator trap potential, $\omega$ the oscillator frequency, $\mu$ the chemical potential and $g$ the strength of interatomic interaction. A positive $g$ correspond to a repulsive interaction and a negative $g$ to an attractive interaction.

Here we shall be interested in the spherically symmetric solution $\Psi(\mathbf{r}) \equiv \psi(r)$ to eq. (0.1) which can be written as

$\left[-\frac{\hbar^{2}}{2 m} \frac{1}{r} \frac{d}{d r} r \frac{d}{d r}+\frac{1}{2} m \omega^{2} r^{2}+g \psi^{2}(r)-\mu\right] \psi(r)=0$.

The ground state of the condensate appears in such a spherically symmetric state. As is Ref. [6], it is convenient to express eq. (0.2) in terms of dimensionless variables defined by $x=r / a$, where $a \equiv \sqrt{\hbar /(m \omega)}$, $\alpha=\mu /(\hbar \omega), \psi(x)=a \sqrt{2 m g} \psi(r) / \hbar$. In terms of these dimensionless variables eq. (0.2) can be written as

$$
\left[-\frac{1}{x} \frac{d}{d x} x \frac{d}{d x}+x^{2}+c \psi^{2}(x)-2 \alpha\right] \psi(x)=0 .
$$

where $c= \pm 1$ carries the sign of $g, c=1$ corresponds to a repulsive interaction and $c=-1$ corresponds to an attractive interaction.

The normalization of the wave function is given by

$$
N=2 \pi \int_{0}^{\infty} d r r \psi^{2}(r)
$$


where $N$ is the total number of atoms in the condensate. In terms of the dimensionless variables defined above, this normalization condition becomes

$$
\int_{0}^{\infty} x d x \psi^{2}(x)=n \equiv \eta N,
$$

where as in Ref. [2] we have introduced a dimensionless coupling $\eta \equiv m g /\left(\pi \hbar^{2}\right)$ of interatomic interactions and a reduced number of particles $n$. From a study of the temperature dependence of the chemical potential for a system of interacting bosons in two dimensions it was concluded in Ref. [2] that a Bose-Einstein-condensate-like behavior is obtained in this system for values of $\eta$ typically smaller than 0.001 . Hence for a qualitative estimate of the number of condensed bosons in this calculation, we shall consider $\eta=0.0001$.

Instead of solving eqs. (0.2) and (0.4), we shall be working with eqs. (0.3) and (0.5). Equation (0.3) is independent of all parameters of the problem, such as, $m$, $\omega, g$, and $N$. The relevant parameters appear in the normalization condition (0.5). The constant $n$ in eq. (0.5) is the reduced number of atoms for the system and is related to the real number of atoms $N$.

Another interesting property of the condensate wave function is its mean-square radius defined by

$$
\left\langle r^{2}\right\rangle=\frac{2 \pi}{N} \int_{0}^{\infty} r^{2} \psi^{2}(r) r d r .
$$

In terms of the dimensionless variables defined above we have

$$
\left\langle x^{2}\right\rangle=\frac{1}{n} \int_{0}^{\infty} x^{2} \psi^{2}(x) x d x .
$$

To solve eq. (0.3) numerically, first we study the asymptotic behavior of its solutions. Since for a sufficiently large $x, \psi(x)$ must vanish asymptotically, the nonlinear term proportional to $\psi^{3}(x)$ can eventually be neglected in eq. (0.3) in the asymptotic region. Thus for large $x$, this equation can be approximated by

$$
\left[-\frac{1}{x} \frac{d}{d x} x \frac{d}{d x}+x^{2}-2 \alpha\right] \psi(x)=0 .
$$

If this equation would be valid for all $x$ this would be the equation for the two-dimensional oscillator in the spherically symmetric state permitting solution for $\alpha=1,3,5, \ldots$ etc. However, in the present problem eq. (0.8) is valid only in the asymptotic region. Considering eq. (0.8) as a mathematical equation valid for all $\alpha$, the asymptotic form of the physically acceptable solution is given by

$$
\lim _{x \rightarrow \infty} \psi(x)=N_{C} \exp \left[-\frac{x^{2}}{2}+(\alpha-1) \ln x\right],
$$

where $N_{C}$ is a normalization constant. The derivative of the wave function in the asymptotic region can be obtained from eq. (0.9) and one obtains the following asymptotic form for the log-derivative of the wave function

$$
\lim _{x \rightarrow \infty} \frac{\psi^{\prime}(x)}{\psi(x)}=\left[-x+\frac{\alpha-1}{x}\right],
$$

which is independent of the normalization constant $N_{C}$ of eq. (0.9) and where the prime denotes derivative with respect to $x$.

Next we consider eq. (0.3) as $x \rightarrow 0$. The nonlinear term approaches a constant in this limit because of the regularity of the wave function at $x=0$. Then one has the following usual conditions

$$
\psi(0)=\text { constant }, \quad \psi^{\prime}(0)=0,
$$

as in the case of the two-dimensional harmonic oscillator problem.

Equation (0.3) is integrated numerically for a given $\alpha$ by the four-point Runge-Kutta rule starting at the origin $(x=0)$ with the initial boundary condition (0.11) with a trial $\psi(0)$. The numerical integration is performed in steps of $d x=\Delta$, where $\Delta$ is typically taken to be 0.0001 . The integration is propagated to $x=x_{\max }$, where the asymptotic condition $(0.10)$ is valid. The agreement between the numerically calculated log-derivative of the wave function and the theoretical result $(0.10)$ was enforced to five significant figures. The maximum value of $x$, up to which we needed to integrate (0.3) numerically for obtaining this precision, is $x_{\max }=5$. If for a trial $\psi(0)$, the agreement of the log-derivative can not be obtained, a new value of $\psi(0)$ is to be chosen. The proper choice of $\psi(0)$ was implemented by the secant method. Even with this method, sometimes it is difficult to obtain the proper value of $\psi(0)$ for a given $\alpha$. Unless the initial guess is "right" and one is sufficiently near the desired solution the method could fail and lead to either the trivial solution $\psi(x)=0$ or an exponentially divergent nonnormalizable solution in the asymptotic region. However, there is one guideline which is of help in finding the proper value of $\psi(0)$. The convergence of the numerical log-derivative to the theoretical result (0.10) with a variation of $\psi(0)$ for a fixed $\alpha$ is monotonic in nature provided that one is near the exact value. If for two trial values of $\psi(0)$ near the exact value one obtains a positive and a negative error for the log-derivative, respectively, the exact $\psi(0)$ lies in between these two trial values.

For both attractive $(c=-1)$ and repulsive $(c=1)$ interatomic interactions, in addition to the ground state solution with no nodes, eq. (0.3) also permits radially excited states with nodes in the wave function. In the absence of the nonlinear term, the spherically-symmetric discrete ground-state solution of the two-dimensional oscillator given by eq. (0.3) is obtained for $\alpha=1$. Radially excited oscillator states appear for $\alpha=3,5, \ldots$ etc. In the presence of the nonlinearity, for attractive interatomic interaction $(c=-1)$, the solutions of the GP equation for the ground state appear for values chemical potential 
$\alpha<1$. For a repulsive interatomic interaction $(c=1)$, these solutions appear for $\alpha>1$. The solutions of eq. (0.3) for the radially excited state with one node for the attractive $(c=-1)$ and repulsive $(c=1)$ cases appear for values of chemical potential $\alpha$ smaller and larger than the energy of the first excited state $(\alpha=3)$ of the harmonic oscillator problem, respectivisuly.
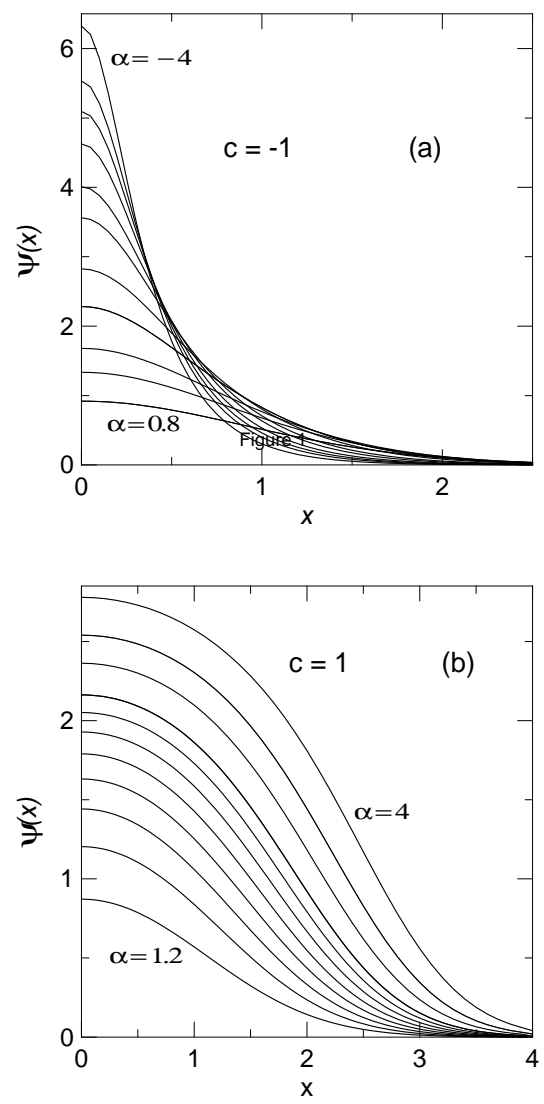

Fig. 1. Ground-state condensate wave function $\psi(x)$ versus $x$ for (a) attractive and (b) repulsive interparticle interactions. The parameters for these cases are given in Table I. The curves appear in the same order as in Table I. The lowermost curve corresponds to the first row in Table I.

First we consider the ground-state solution of eq. (0.3) for different $\alpha$ in the cases of both attractive and repulsive interactions. The relevant parameters for these solutions (values of the wave-function at the origin $\psi(0)$, reduced number $n$, and mean-square radii $\left.\left\langle x^{2}\right\rangle\right)$ are listed in table 1 . The wave functions for different values of $\alpha$ for the attractive and repulsive interparticle interactions for the cases shown in table 1 are exhibited in figures 1(a) and $1(\mathrm{~b})$, respectively, where we plot $\psi(x)$ versus $x$. The curves in figures 1(a) and 1(b) appear in the same order as the rows in table 1 and it is easy to identify the corresponding values of $\alpha$ from the values of $\psi(0)$ of each curve. From figures 1(a) and (b) we find that the na- ture of the wave function for these two cases are quite different.

Table 1: Parameters for the numerical solution of the GP equation (0.3) for $c= \pm 1$ for the ground state wave function. The first four columns refer to the attractive interaction $c=-1$ and the last four columns refer to the repulsive interaction $c=1$.

\begin{tabular}{|c|c|c|c|c|c|c|c|}
\hline$\alpha$ & $\psi(0)$ & $n$ & $\left\langle x^{2}\right\rangle$ & $\alpha$ & $\psi(0)$ & $n$ & $\left\langle x^{2}\right\rangle$ \\
\hline 1.0 & 0 & 0 & 0 & 1.0 & 0 & 0 & 0 \\
0.8 & 0.9185 & 0.3663 & 0.9030 & 1.2 & 0.8719 & 0.4353 & 1.1027 \\
0.6 & 1.3347 & 0.6690 & 0.8127 & 1.4 & 1.2036 & 0.9435 & 1.2103 \\
0.4 & 1.6795 & 0.9147 & 0.7297 & 1.6 & 1.4415 & 1.5276 & 1.3219 \\
0.0 & 2.2827 & 1.2655 & 0.5872 & 1.8 & 1.6308 & 2.1894 & 1.4368 \\
-0.4 & 2.8255 & 1.4798 & 0.4757 & 2.0 & 1.7896 & 2.9303 & 1.5544 \\
-1.0 & 3.5624 & 1.6530 & 0.3564 & 2.2 & 1.9276 & 3.7509 & 1.6741 \\
-1.4 & 4.0097 & 1.7152 & 0.3005 & 2.4 & 2.0507 & 4.6518 & 1.7956 \\
-2.0 & 4.6249 & 1.7695 & 0.2400 & 2.6 & 2.1627 & 5.6331 & 1.9186 \\
-2.5 & 5.0942 & 1.7957 & 0.2040 & 3.0 & 2.3626 & 7.8377 & 2.1679 \\
-3.0 & 5.5307 & 1.8126 & 0.1767 & 3.4 & 2.5401 & 10.3651 & 2.4206 \\
-4.0 & 6.3252 & 1.8319 & 0.1385 & 4.0 & 2.7786 & 14.7609 & 2.8041 \\
\hline
\end{tabular}

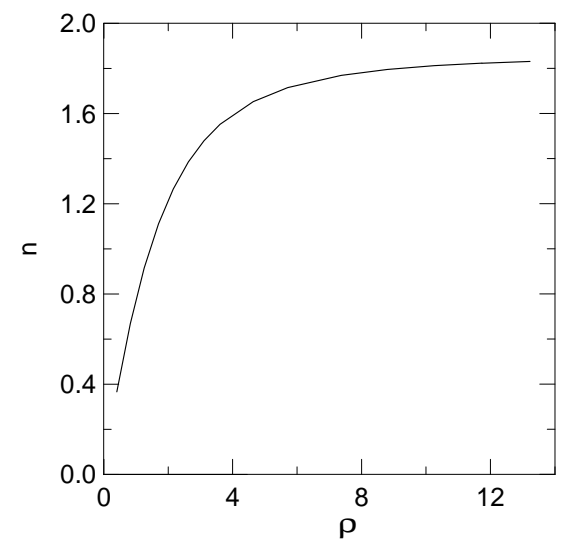

Fig. 2. The reduced number of particles $n$ versus density $\rho \equiv n /\left\langle x^{2}\right\rangle$ of the ground-state condensate for the attractive interaction.

Table 2: Parameters for the numerical solution of the GP equation (0.3) for $c= \pm 1$ for the wave function of the first excited state. The first four columns refer to the attractive interaction $c=-1$ and the last four columns refer to the repulsive interaction $c=1$.

\begin{tabular}{|c|c|c|c|c|c|c|c|}
\hline$\alpha$ & $\psi(0)$ & $n$ & $\left\langle x^{2}\right\rangle$ & $\alpha$ & $\psi(0)$ & $n$ & $\left\langle x^{2}\right\rangle$ \\
\hline 3.0 & 0 & 0 & 0 & 3.0 & 0 & 0 & 0 \\
2.9 & 0.9071 & 0.3940 & 2.9505 & 3.1 & 0.8814 & 0.4064 & 3.0505 \\
2.8 & 1.3012 & 0.7769 & 2.9012 & 3.2 & 1.2278 & 0.8254 & 3.1013 \\
2.6 & 1.8898 & 1.5118 & 2.8039 & 3.6 & 2.0006 & 2.6466 & 3.3101 \\
2.0 & 3.1912 & 3.5069 & 2.5215 & 4.0 & 2.4347 & 4.7325 & 3.5282 \\
1.5 & 4.0643 & 4.9560 & 2.2976 & 4.5 & 2.7895 & 7.7573 & 3.8124 \\
\hline
\end{tabular}

For the attractive interparticle interaction, the wave function is more sharply peaked at $x=0$ than in the case of the repulsive interparticle interaction. Consequently, 
in the attractive case one has smaller values of the reduced number $n$ and mean square radii $\left\langle x^{2}\right\rangle$ of the wave function. For the attractive case we find from table 1 that with a reduction of the chemical potential $\alpha$ the reduced number $n$ increases slowly and the mean square radius $\left\langle x^{2}\right\rangle$ decreases rapidly, so that the density of the condensate $\rho \equiv n /\left\langle x^{2}\right\rangle$ tends to diverge as $n$ tends to a maximum value $n_{\max }$. This means that there is a maximum number of particles in the condensate in this case. This peculiar behavior in the attractive case is demonstrated in figure 2 where we plot the reduced number $n$ of the condensate in the ground state versus the density $\rho$. From figure 2 we find that with the reduction of the chemical potential, the reduced number of particles $n$ in the condensate attains a saturation value. Numerically, we find this value to be

$$
n_{\max } \equiv \eta N_{\max } \approx 1.85
$$

However, during this process the mean square radius continues to decrease thus leading to a divergence of the density of the condensate. For $n>n_{\max }$, there is no stable solution of the GP equation. There is no such limit on $n$ in the repulsive case. In that case with the increase of the chemical potential $\alpha$ the condensate increases in size as the number of particles in the condensate increases. These behaviors of the Bose-Einstein condensate in two dimensions were also noted in three dimensions 11.13 .

Next we consider solutions to eq. (0.3) with radial excitation. The present numerical method is equally applicable to the ground and all radially excited states of the condensate. In this work we shall consider excited solutions with only one node for both attractive and repulsive interparticle interactions, which we discuss next. In table 2 we exhibit the parameters of some of such solutions. In figures $3(\mathrm{a})$ and $3(\mathrm{~b})$ we plot the respective wave functions $\psi(x)$ versus $x$ for the attractive and repulsive interactions. We again find that in the case of attractive interaction the wave function is narrowly peaked near $x=0$, and for repulsive interaction it is more extended in space to larger values of $x$. Consequently, the reduced number $n$ and mean square radii $\left\langle x^{2}\right\rangle$ are larger in the case of repulsive interaction. We again find from table 2 that in the attractive case, with a reduction of the chemical potential $\alpha$, the mean square radius decreases as the reduced number $n$ increases. It is expected that there should be a saturation on $n$ in this case also. However, we did not try to find this saturation numerically, which should occur for values of $\alpha$ much smaller than those presented in table 2 leading to a much larger value of $n_{\max }$ than in eq. (0.12).

Although, we have considered the problem in a system of dimensionless units, it is interesting to see what our results correspond to in actual units. If we consider the typical value 0.0001 of $\eta$ favorable to the formation of Bose-Einstein condensate [2] as commented after eq. (0.5), we can calculate the actual numer of atoms $N$. The number of atoms $N$ in the condensate for all cases reported in tables 1 and 2 is maximum for the ground state of the repulsive interatomic interaction for $\alpha=4$. This number in this case is $N=147,600$. If we consider a trap frequency such that $\sqrt{\hbar /(m \omega)}=10,000 \AA$ [5], then the root-mean-square radius for the above condensate with 147,600 atoms is $16,700 \AA$. In the attractive case the maximum numer of particles given by eq. (0.12) becomes $N_{\max }=18,500$. Both the size and numbers of particles seem to be very reasonable for the condensate [3,5].

In this work we have investigated the numerical solution of the Gross-Pitaevskii equation (0.1) for BoseEinstein condensation in two dimensions under the action of a harmonic oscillator trap potential for bosonic atoms interacting via both attractive and repulsive interparticle interactions. In both cases we considered the wave function for the ground state and radially excited state with one node. We expressed the GP equation in dimensionless units independent of all parameters, such as, atomic mass, harmonic oscillator frequency, number of atoms in the condensate, and strength of atomic interaction. The relevant parameters appear in the normalization condition (0.5) of the wave function. We derive the boundary conditions (0.10) and (0.11) of the solution of the dimensionless GP equation (0.3), which is integrated from the origin outwards in steps of 0.0001 by the four-point Runge-Kutta rule consistent with the boundary condition. At a particular value of the chemical potential, the correct solution is obtained after a proper guess of the boundary condition of the wave function at the origin. From a initial trial value of the wave function at the origin, Newton-Raphson method is used to obtain the correct wave function after a matching with the boundary condition in the asymptotic region. In both the ground and excited states it is found that the wave function is sharply peaked near the origin for attractive interatomic interaction. For a repulsive interatomic interaction the wave function extends over a larger region of space. In the case of an attractive potential, the mean square radius decreases with an increase of the number of particles in the condensate. Consequently, a stable solution of the GP equation can be obtained for a maximum number of particles in the condensate. For the ground state the maximum reduced number of particles is given by eq. (0.12). For the repulsive case there is no such limit on the number of particles in the condensate.

We thank the authors of Ref. [6] for a copy of their work prior to publication. The work is supported in part by the Conselho Nacional de Desenvolvimento Científico e Tecnológico and Fundação de Amparo à Pesquisa do Estado de São Paulo of Brazil.

[1] M. H. Anderson, J. R. Ensher, M. R. Mathews, C. E. 
Wieman, and E. A. Cornell, Science 269 (1995) 198; C. C. Bradley, C. A. Sackett, and R. G. Hulet, Phys. Rev. Lett. 78 (1997) 985; J. R. Ensher, D. S. Jin, M. R. Mathews, C. E. Wieman, and E. A. Cornell, Phys. Rev. Lett. 77 (1996) 4984; K. B. Dadic, M. O. Mewes, M. R. Andrews, N. J. van Druten, D. S. Durfee, D. M. Kurn, and W. Ketterle, Phys. Rev. Lett. 75 (1995) 3969; D. G. Fried, T. C. Killian, L. Willmann, D. Landhuis, S. C. Moss, D. Kleppner, T. J. Greytak, Phys. Rev. Lett. 81 (1998) 3811.

[2] M. Bayindir and B. Tanatar, Phys. Rev. A 58 (1998) 3134.

[3] M. Edwards and K. Burnett, Phys. Rev. A 51 (1995) 1382.

[4] P. A. Ruprecht, M. J. Holland, K. Burnett, and M. Edwards, Phys. Rev. A 51 (1995) 4704; M. Edwards, P. A. Ruprecht, K. Burnett, R. J. Dodd, and C. W. Clark, Phys. Rev. Lett. 77 (1996) 1671.

[5] F. Dalfovo and S. Stringari, Phys. Rev. A 53 (1996) 2477, S. Giorgini, L. P. Pitaevskii, and S. Stringari, Phys. Rev. A 54 (1996) R4633.

[6] A. Gammal, T. Frederico, and L. Tomio, Phys. Rev. E 60 (1999) 2421.

[7] E. A. Hinds, M. G. Boshier, and I. G. Hughes, Phys. Rev. Lett. 80 (1998) 645.

[8] E. P. Gross, Nuovo Cimento 20 (1961) 454; L. P. Pitaevskii, Zh. Eksp. Teor. Fiz. 40 (1961) 646 [Sov. Phys. JETP 13 (1961) 451].

[9] P. C. Hoenberg, Phys. Rev. 158 (1967) 383.

[10] V. Bagnato and D. Kleppner, Phys. Rev. A 44 (1991) 7439.

[11] F. Dalfovo, S. Giorgini, L. P. Pitaevskii, and S. Stingari, Rev. Mod. Phys. 71 (1999) 463.

[12] J. J. Rehr and N. D. Mermin, Phys. Rev. B 1 (1970) 3160; R. Masut and W. J. Mullin, Am. J. Phys. 47 (1979) 493; H. A. Gersch, J. Chem. Phys. 27 (1957) 928.

[13] R. J. Dodd, M. Edwards, C. J. Williams, C. W. Clark, M. J. Holland, P. A. Ruprecht, and K. Burnett, Phys. Rev. A 54, (1996) 661. 